\title{
Contemplación Humana y Placer Divino EN ARISTÓteles
}

\author{
Manuel Cruz, Ortiz de Landázuri \\ (Universidad de Navarra)
}

\section{Introducción}

¿Cómo se debe entender la afirmación de Aristóteles en la Metafisi$c a$, cuando dice que la actividad de Dios es también placer? Como se verá, se trata de un texto en el que declara sin ambages que la existencia del entendimiento que se entiende a sí mismo es sumamente placentera. ¿Se puede decir que Dios disfruta? Aristóteles, en la Metafísica, afirma que la actividad divina es puro placer, porque su actividad es perfecta ${ }^{1} \mathrm{y}$, por otro lado, vincula el placer en la Ética a Nicómaco con la perfección de las actividades, poniendo además como ejemplo las actividades cognoscitivas ${ }^{2}$. Pero, además, si es posible decir que Dios disfruta, o que hay placer en la contemplación, habrá que ver cómo es posible que haya un placer "espiritual", desvinculado del cuerpo puesto que, como se afirma en el De Anima, la actividad de entender está más allá de lo meramente corporal $^{3}$. Por otro lado, habrá que ver si se da en el caso del ser humano un placer "espiritual", puesto que la actividad contemplativa asemeja a la actividad divina ${ }^{4}$ y no se encuentra limitada por lo material.

${ }^{1}$ Cfr. Met. A.7, 1072 b 14-16. Para la Metafísica empleo la traducción de V. García Yebra (Gredos, Madrid, 1970) con algunas ligeras variaciones.

${ }^{2}$ Cfr. Eth. Nic. X.4, 1174 b 14-24. Para la Ética a Nicómaco empleo la traducción de M. Araujo y J. Marías (Centro de estudios políticos y constitucionales, Madrid, 1999) con algunas ligeras variaciones.

${ }^{3}$ Cfr. De Anima III.4, 429 b 5-6. Para el De Anima empleo la traducción de T. Calvo (Gredos, Madrid, 1978) con algunas ligeras variaciones.

4 "Insofern ist die Noesis Noeseos eine Aktivität, die nicht nur Gott zukommt. Das göttliche und das menschliche noetische Denken sind zwar nicht identisch, sondern verschieden, aber sie sind nicht absolut verschieden. Sie stehen im Verhältnis der 


\section{Dios también disfruta}

Dios, como pura actividad, como pensamiento que se piensa a sí mismo, encuentra el máximo deleite en esta actividad. La existencia del acto puro es "como la mejor para nosotros durante corto tiempo (pues aquel ente siempre es así; para nosotros, en cambio, esto es imposible),

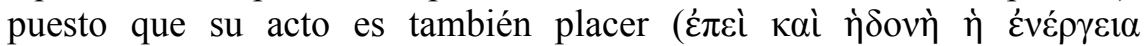

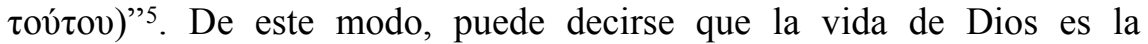

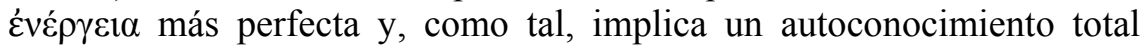
máximamente placentero:

"El entendimiento se entiende a sí mismo por captación de lo inteligible; pues se hace inteligible estableciendo contacto y entendiendo, de suerte que entendimiento e inteligible se identifican. Pues el receptáculo de lo inteligible y de la sustancia es entendimiento, y está en acto teniéndolos, de suerte que esto más que aquello es lo divino que el entendimiento parece tener, y la contemplación es lo más agradable y lo más noble. Si, por consiguiente, Dios se halla siempre tan bien como nosotros algunas veces, es cosa admirable; $y$, si se halla mejor, todavía más admirable. Y así es como se halla. Y tiene vida, pues el acto del entendimiento es

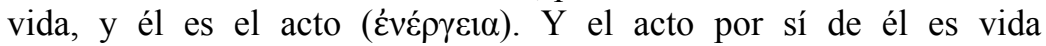
nobilísima y eterna"6.

Aristóteles pone de manifiesto en qué consiste la actividad divina: una autoconocimiento pleno $^{7}$, una experiencia total de sí mismo (’̀

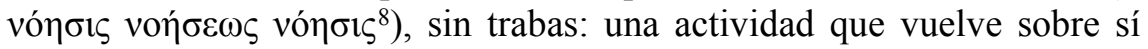

Analogie". OEHLER, K., Subjektivität und Selbstbewußtsein in der Antike, Königshausen \& Neumann, Würzburg, 1997, p. 58. También cfr. NATALI, C., "Attività di Dio e dell'uomo nella Metafisica", Rivista di filosofia neo-escolastica 85 (1993), pp. 344-345.

${ }^{5}$ Met. $\Lambda .7,1072$ b 14-16.

${ }^{6}$ Met. $\Lambda .7,1072$ b 19-28.

${ }^{7}$ Algunos autores han considerado un tanto absurda la idea de que la actividad de Dios sea su propio autoconocimiento. Cfr. Ross, W., Aristotle's Metaphysics, Clarendon Press, Oxford, 1924, pp. cxli-cxlii; NORMAN, R. “Aristotle's Philosopher-God”, Phronesis 14 (1969), pp. 63-74; Randall, J. H., Aristotle, Columbia University Press, New York, 1960, pp. 135-136. Frente a éstos Dudley ha replicado a muchos de los argumentos: DudLeY, J., Gott und $\theta \varepsilon \omega \rho i \alpha$ bei Aristoteles, Peter Lang, Frankfurt am Main/Bern, 1982, pp. 92-104.

${ }^{8}$ Met. $\Lambda .9,1074$ b 34. Comenta Edler al respecto: "The expression is very well chosen and serves the purpose to state that (a) there is no distinction between the thinking subject and its object; (b) that there is no distinction between the thinking subject and 
misma sin impedimento y, que a la vez, es placentera. ¿Cuál es la conexión entre la actividad divina y su placer correspondiente? Y, más aún, ¿hay alguna relación entre el placer de la actividad divina de contemplación y el placer de la contemplación humana? La cuestión puede quedar más clara si se examina el vínculo que establece Aristóteles entre placer y

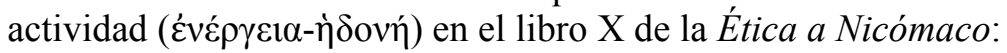

"Puesto que todo sentido actúa con relación a su objeto, y lo hace perfectamente el que está bien dispuesto hacia lo más excelente que por él puede ser percibido (en esto parece consistir, en efecto, la

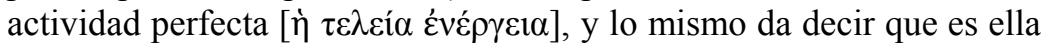
la que actúa o el órgano en que se da), en cada sentido será la mejor la actividad del órgano que esté mejor dispuesto respecto de lo más excelente que cae bajo su radio de acción, y esta actividad será a la vez la más perfecta y la más agradable. Pues si bien toda actividad va acompañada de placer, e igualmente todo pensamiento $\mathrm{y}$ contemplación, es más agradable la más perfecta, y es la más perfecta la del órgano bien dispuesto respecto de lo mejor que cae bajo su radio de acción, y el placer perfecciona la actividad"9.

La doctrina del placer del libro X de la Ética a Nicómaco parece te-

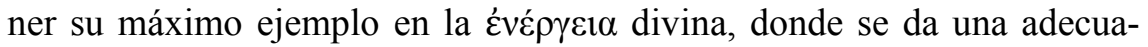
ción plena entre el cognoscente y lo conocido, de donde brota una acción de conocimiento perfecta, sin trabas. El placer acompaña a la actividad perfecta cognoscitiva pero, si Dios disfruta, el placer no puede ser, en ningún caso, una perfección añadida, ni una sensación recibida, puesto que a Dios, como acto perfecto, no le puede faltar nada. Por tanto, el placer es un "sentirse" total, inherente a la actividad de autoconocimiento, y que no implica pasividad o potencialidad.

¿Cómo es esta actividad de Dios, sumamente placentera? ¿En qué consiste exactamente el pensamiento que se piensa a sí mismo, la autoconciencia absoluta? Evidentemente, es imposible saberlo, pero cabe decir que es una actividad perfecta, y por lo tanto "libre de trabas". Se trata de un conocimiento que se experimenta a sí mismo sin impedimentos, sin mediaciones, lo cual arroja algo de luz sobre en qué debe consistir la felicidad humana, porque la actividad más plena no consiste tanto en una produc-

mind on the one hand and its thinking on the other hand; (c) that the first being is pure activity. The expression 'thinking of thinking' is more precise than 'the first being thinks itself', for in the latter formulation the distinction between the ontological order

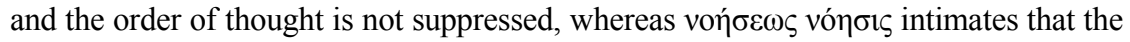
very being of the first principle is thinking, and thinking is being". ELDER, L., Aristotle's Theology, Van Gorcum, Assen, 1972, pp. 259-260.

${ }^{9}$ Eth. Nic. X.4, 1174 b 14-24. 


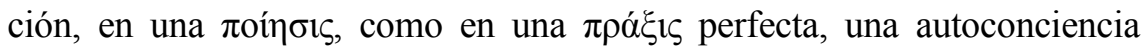
libre de trabas, el experimentar la propia vida sin problemas de ningún género, sin preocupaciones. A esto se le opone rotundamente la vida preocupada y la angustia ante la nada. La vida como preocupación lleva a vivir fuera de sí, en las cosas, sin permitir esa experiencia plena de la propia vida, a la que no le falta nada; la angustia, es más bien la incapacidad de autoconciencia por la desazón ante el mundo, como diría Heidegger, es la nada que se patentiza ${ }^{10}$. La angustia es la incapacidad de experimentar el propio ser en plenitud, la problematización de la propia existencia. Justamente la actividad divina, al ser la actividad pura, es a la vez una experiencia máximamente feliz, sin trabas, sin preocupaciones: la autoconciencia

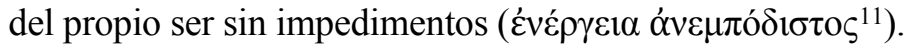

\section{Placer y motor inmóvil}

Un punto interesante que merece la pena considerar es la relación que guarda la doctrina del acto puro con el pensamiento de Eudoxo. Según la postura de Schadewaldt ${ }^{12}$ parece que fue Eudoxo quien identificó la idea de bien platónica con el placer, uniendo el placer a la actividad. Si Dios es la suma actividad, debe por tanto ser también el sumo placer, y aquello que, siendo objeto de deseo para todos los seres, mueve sin ser movido. Ya que todos persiguen el placer, y que todos se mueven hacia la actividad perfecta, todos aspiran llegar a Dios, que es el sumo disfrute, quien colma todos los deseos con su actividad perfecta. Así habría llegado Eudoxo a una curiosa relación entre placer, actividad y objeto de de-

10 “Solemos decir que en la angustia "uno está desazonado”. ¿Qué quiere decir este "uno"? No podemos decir de qué le viene a uno esta desazón. Nos encontramos así, y nada más. Todas las cosas como nosotros mismos se sumergen en una indiferenciación. Pero no como si fuera un mero desaparecer, sino como un alejarse que es un volverse hacia nosotros. Este alejarse el ente en total, que nos acosa en la angustia, nos oprime. No queda asidero ninguno. Lo único que queda y nos sobrecoge al escapársenos el ente es este "ninguno". La angustia hace patente la nada. Estamos "suspensos" en angustia. Más claro, la angustia nos deja suspensos porque hace que se nos escape el ente en total. Por esto sucede que nosotros mismos estos hombres que somos-, estando en medio del ente, nos escapemos de nosotros mismos. Por esto, en realidad, no somos "yo" ni "tú" los desazonados, sino "uno". Sólo resta el puro existir en la conmoción de ese estar suspenso en que no hay nada donde agarrarse”. HeidegGer, M., ¿Qué es metafísica?, Editorial Siglo XX, Buenos Aires, 1986, pp. 70-71.

${ }^{11}$ Eth. Nic. VII.12 1153 a 14-15.

12 Cfr. SchadewaldT, W., "Eudoxos von Knidos und die Lehre vom unbewegten Beweger”, Satura. Früchte aus der antiken Welt, K. F. Stroheker (ed.), Verlag für Kunst und Wissenschaft, Baden-Baden, 1952, pp. 101-129. 
seo, que le habría hecho concebir a Dios como bien absoluto, acto puro, motor inmóvil y, sobre todo, máxima experiencia hedónica.

Sea o no algo más que una hipótesis, cabe preguntarse si hay un esquema semejante en la filosofía de Aristóteles. Hay varios textos que apuntan a esto. En los libros VII y X de la Ética a Nicómaco Aristóteles parece dar por válida la postura de Eudoxo de que el placer debe ser un bien, porque es aquello hacia lo que todos los seres tienden, y su contrario, el dolor, es rechazado por todos ${ }^{13}$. En la Metafisica Aristóteles trata a Dios como el acto puro, aquello que mueve sin ser movido ("mueve en cuanto que es amado, mientras que todas las demás cosas mueven al ser movidas" ${ }^{14}$ ) y, más aún, identifica, como se ha visto, la actividad de Dios con el placer: "puesto que su acto es también placer"15. Además, un movimiento de todos los seres hacia la causa final por medio del deseo (ya que la causa final es placentera) resultaría encajar con un punto importante de la ética del Estagirita, a saber, que la actividad más alta en el ser humano es la contemplación de Dios, siendo éste el objeto más deseable y pleno para el conocimiento que, aunque no se afirma de modo explícito en la Ética a Nicómaco ${ }^{16}$, se expresa al final de la Ética a Eudemo:

"Esta elección y adquisición de bienes naturales -bienes del cuerpo, riquezas, amigos y otros bienes- que más promueve la contemplación

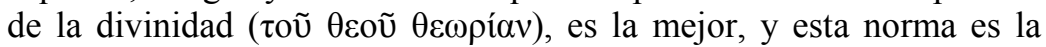
más bella" 17 .

Por otro lado, hay un texto en el De Motu Animalium que parece apuntar en esta dirección:

"El bien eterno, el bien verdadero y primero y que no existe una vez sí y otra no, es demasiado divino y estimable como para que haya algo anterior. Por tanto, lo primero mueve sin ser movido, mientras que el deseo y lo deseable mueven después de haber sido movidos ( $\tau$ ò $\mu \grave{v} v$

${ }^{13}$ Cfr. Eth. Nic. VII.13, 1153 b 1-9; X.2, 1172 b 9-1173 a 14.

14 Met. $\Lambda .7,1072$ b 3-4.

15 Met. $\Lambda .7,1072$ b 16.

${ }^{16}$ De modo implícito sí hay alguna referencia, como por ejemplo ésta: "si, por tanto, la mente es divina respecto del hombre también la vida según ella es divina respecto de la vida humana. Pero no hemos de tener, como algunos nos aconsejan, pensamientos humanos puesto que somos hombres, ni mortales puesto que somos mortales, sino en la medida de lo posible vivir inmortalizándonos y hacer todo lo que está a nuestro alcance por vivir de acuerdo con lo más excelente que hay en nosotros". Eth. Nic. X.7, 1177 b 30-35. Parece que la ética tiene como fin la contemplación, lo cual sitúa al hombre en un plano divino.

17 Ética a Eudemo VII.15, 1249 b 16-19. 


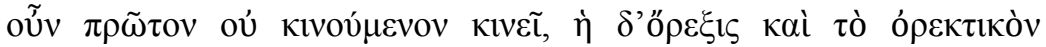

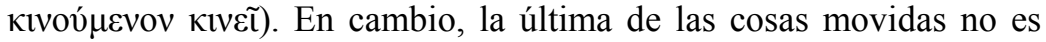
necesario que mueva nada" 18 .

Aquí parece que se establece una conexión entre el bien, entendido como motor inmóvil (mueve sin ser movido) y el deseo (mueve siendo a la vez movido). De este modo podría desprenderse que Dios, el acto puro, es objeto de deseo, aquello hacia lo que los seres tienden. Sin embargo, Aristóteles no llega a afirmar tanto y, en este caso, sólo cabe moverse en el terreno de las hipótesis. Aristóteles nunca dice que Dios mueva a todos los seres siendo el objeto de sus deseos (y por lo tanto su bien), ni que sea su máxima actividad y el placer más pleno para los seres. Aristóteles sólo afirma que Dios se goza en su propia actividad: que él es, en sí mismo y para sí mismo, la máxima actividad y el máximo placer, y que mueve sin ser movido ${ }^{19}$.

Aunque Aristóteles aprecie de manera considerable los argumentos de Eudoxo, no parece deducir de éstos que el placer sea el bien supremo (como lo creía Eudoxo) sino tan sólo uno de los bienes. De este modo, el Estagirita recoge la crítica de Platón al placer como bien supremo y parece tenerla por válida:

"La vida agradable es más apetecible con sabiduría que sin ella, y si la mezcla es mejor, el placer no es el bien, porque el bien no puede hacerse más apetecible por añadírsele nada" ${ }^{20}$.

El placer es un bien, y en este punto Eudoxo tenía razón, pero de ahí no se puede deducir que sea el Bien, puesto que por sí solo no es lo mejor. De alguna manera cabe admitir que para Aristóteles el acto puro, la causa final, mueve sin ser movido, es lo más deseable y su actividad es también puro placer. Por otro lado, como se verá, al ser lo más separado

${ }^{18}$ De Motu Animalium 6, 700 b 33-701 a 6.

${ }^{19} \mathrm{El}$ acto puro mueve en cuanto que es amado y deseado: esto no quiere decir que sea un placer que persiguen todos los seres naturales, sino más bien que los seres imitan el movimiento del acto puro, tratan de hacerse semejantes a él porque en la medida en que el acto es más perfecto la existencia es más dichosa: "How, we may ask, does love or desire for the prime mover produce the physical movements that have to be explained? The theory is that each of these unities of soul and body desires a life as like as possible to that of its moving principle. The life of its moving principle is a continuous unchanging activity of pure thought (with addition, we may suppose, in the case of the intelligences, of love of the prime mover). The spheres cannot reproduce this, but they do the next best by performing the only perfectly continuous physical movement, movement in a circle”. Ross, W., Aristotle's Metaphysics, Clarendon Press, Oxford, 1924, pp. cxxxvii-iii.

${ }^{20}$ Eth. Nic. X.2, 1172 b 29-31. 
de lo material su contemplación resultaría máximamente perfecta, y por lo tanto placentera. Ahora bien, también es cierto que Aristóteles tiene sus reticencias a admitir que el bien supremo sea el placer. Dios es ante todo la actividad perfecta, y secundariamente a esa actividad, es también placer. Por otro lado, tampoco hay razones de peso para admitir que aquello hacia lo que todos se dirigen sea Dios (el primer motor que mueve sin ser movido).

\section{Naturaleza de la contemplación}

El placer divino es inaccesible para el ser humano, pero parece posible aproximarse a él en la medida en que el hombre conoce ${ }^{21}$. En el caso del ser humano la contemplación $(\theta \varepsilon \omega \rho i ́ \alpha)$ asemeja a la actividad divina $^{22} \mathrm{y}$, además, la consideración del placer divino arroja luz para entender el placer intelectual en el caso del ser humano, pues ésta no es una actividad material. En la doctrina del placer del libro X de la Ética a Nicómaco Aristóteles vincula el placer con los actos cognoscitivos y, especialmente, con la contemplación.

¿Qué dice Aristóteles en el De Anima sobre el conocimiento intelectual? Básicamente, el intelecto es aquello con lo que el alma razona

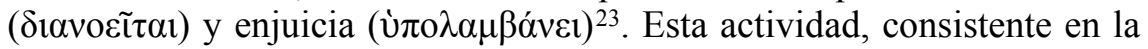
aprensión de formas, no tiene naturaleza propia, pues no es ninguno de los entes antes de entender ${ }^{24}$, y es así impasible y separable de la materia $^{25}$. Se trata, por tanto, de una actividad que va más allá de la sensibilidad, ya que, mientras los sentidos se limitan a presentar objetos (cualidades), mediante el intelecto se comprende el objeto, se entiende qué es:

"Con la facultad sensitiva discierne lo caliente y lo frío así como aquellas cualidades de las que la carne constituye una cierta

${ }^{21}$ La diferencia de placeres reside en la perfección de la actividad. Dios vuelve plenamente sobre sí mismo, mientras que el ser humano sólo contempla lo exterior a él. "Gott kann kontinuerlich sich selbst denken, ohne vorher fremde Gegenstände aufnehmen zu müssen. [...] Der Mensch ist also auch in seinem höchsten kognitiven Vollzug letzlich auf die Welt und damit auf «das Andere» angewiesen". HerzBerG, S., "Theoretische Lebensform und Natur des Menschen bei Aristoteles", Theologie und Philosophie 86 (2011), p. 11.

22 Burnyeat incluso afirma que es incluso más que la imitación de la actividad divina, una participación en ella. Cfr. BuRnYeAt, M. F., Aristotle's Divine Intellect, Marquette University Press, Milwaukee, 2008, p. 43.

${ }^{23}$ Cfr. De Anima III.4, 429 a 22-23.

${ }^{24}$ Cfr. De Anima III.4, 429 a 24-28.

${ }^{25}$ Cfr. De Anima III.4, 429 b 5-6. 
proporción combinatoria; en cuanto a la esencia de la carne, la discierne ya con otra facultad separada, ya con la misma facultad, siendo ésta respecto de sí misma lo que la línea curva es respecto de sí misma una vez enderezada" 26 .

Comprender un objeto es entender qué es, captar su esencia ${ }^{27}$. A través de las cualidades presentadas por los sentidos, el intelecto capta el ser de la cosa, la sustancialidad que se nos hace presente a través de las cualidades.

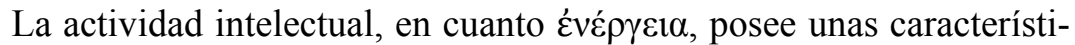
cas que la hacen muy distinta de cualquier otro tipo de actividad. Esto es de suma importancia para entender el placer intelectual, ya que el placer acompaña a la actividad y depende cualitativamente de ella. A diferencia de la actividad sensitiva, la actividad intelectual no se encuentra afectada por la pasibilidad, es $\alpha \dot{\alpha} \alpha \theta \dot{s}$. La pasividad presente en la sensibilidad consiste en que necesita de un estímulo externo para llegar al acto: si bien es acto del alma ${ }^{28}$, a la vez precisa de un elemento exterior en acto que actualice la capacidad cognoscitiva ${ }^{29}$. Sin embargo, a diferencia de ésta, tenemos la impasibilidad de la actividad intelectual:

"Resulta evidente que la impasibilidad de la facultad sensitiva y la de la facultad intelectiva no son del mismo tipo: el sentido, desde luego, no es capaz de percibir tras haber sido afectado por un objeto fuertemente sensible, por ejemplo, no percibe el sonido después de sonidos intensos, ni es capaz de ver u oler, tras haber sido afectado por colores u olores fuertes; el intelecto, por el contrario, tras haber entendido un objeto fuertemente inteligible, no entiende menos sino más, incluso, los objetos de rango inferior" ${ }^{30}$.

26 De Anima III.4, 429 b 15-18.

${ }^{27}$ Esta comprensión de la esencia es explicada a menudo como la posesión de la forma sin la materia. Lo que en acto es indivisible, la inteligencia lo separa y comprende: "L'intellect, en séparant la forme de la matière, pense comme divisé ce qui est en réalité indivisible, de même que le point qui unit les deux segments de la ligne brisée est à fois unique et double". RODIER, G., Commentaire sur le "Traité de l'âme" d'Aristote, Vrin, Paris, 1900, p. 448. Sin embargo no se debe perder de vista que esta comprensión no es una "extracción” de la forma, sino una posesión intencional de ésta, presente en la cosa misma.

${ }^{28}$ La capacidad de recibir formas a través de la materialidad se debe a la acción del alma. Por eso, conocer a nivel sensible es un acto profundamente anímico: "La potentialité dynamique et réceptrice de la faculté sensible n'est telle qu'en raison de sa plasticité et de sa spiritualité". DE CORTE, M., La doctrine de l'intelligence chez Aristote, Vrin, Paris, 1934, p. 149.

${ }^{29}$ Cfr. De Anima II.5, 417 b 19-21.

${ }^{30}$ De Anima III.4, 429 a 30-429 b 5. 
La actividad intelectual es más perfecta que la sensitiva o, si se prefiere, es más actividad. Mientras el conocimiento sensible viene limitado por la potencia propia del órgano, por la materialidad, la actividad intelectual no tiene propiamente limitación material ("puesto que entiende todas las cosas, necesariamente ha de ser sin mezcla" 31 ), y el ejercicio perfecto de la actividad permite que ésta se realice posteriormente con más perfección: la actividad misma del conocimiento intelectual es cada vez más perfecta, a medida que se ejercita ${ }^{32}$.

Esta actividad no viene determinada por una naturaleza, de manera que el intelecto puede conocer, en principio, cualquier $\operatorname{cosa}^{33}$. La actividad

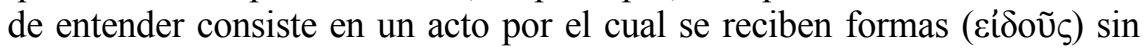
que el entender mismo se identifique con tales formas, al ser el intelecto (voũs) impasible ${ }^{34}$. Por tanto, afirma el Estagirita que "no tiene naturaleza alguna propia aparte de su misma potencialidad"35. Si Aristóteles concibe la actividad intelectual como la más perfecta que hay en el hombre y la que más se puede ejercer ${ }^{36}$, es porque en definitiva no se encuentra limitada por la materia $\mathrm{o}$, si se prefiere, por una naturaleza material. A diferencia de la sensibilidad, que conoce desde un órgano, la actividad intelectual, en cuanto tal, no posee una naturaleza desde la que entender, "ya que lo que exhibe su propia forma obstaculiza e interfiere a la ajena" 37 .

Así pues, se trata de la actividad más perfecta que el alma humana puede llevar a cabo: mientras que en el nivel vegetativo y sensitivo las actividades se encuentran limitadas por la materia, por la potencialidad, en el nivel intelectual la actividad, de suyo, es plena, sólo encuentra impedi-

31 De Anima III.4, 429 a 18.

${ }^{32}$ La actividad intelectual, a medida que se ejercita, es objetivamente más perfecta: se conoce cada vez mejor; a la vez, el placer concomitante a la actividad, hace que esa actividad se realice subjetivamente mejor, que aquel que conoce encuentre agrado al realizarlo, y así se vincule de manera más perfecta con aquello que conoce: "los placeres que resultan de pensar y aprender nos harán pensar y aprender más" Eth. Nic. VII.12, 1153 a 23-24.

33 Polansky remarca que Aristóteles trata a la inteligencia como una posibilidad pura, en vez de una potencia (como sería el caso de la sensibilidad): "Speaking of mind

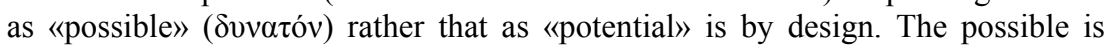
broader than and encompasses potentiality and power. [...] Whether mind knows nothing, something or everything, it nevertheless has at least the possibility of thinking everything at all'. POLAnSKy, R., Aristotle's De Anima, Cambridge University Press, New York 2007, p. 439.

${ }^{34}$ Cfr. De Anima III.4 429 a 15-17.

35 De Anima III.4 429 a 21-22.

${ }^{36}$ Cfr. Eth. Nic. X.7, 1177 a 21-22.

37 De Anima III.4, 429 a 20-21. 
mento en que para realizarse depende de la actividad previa de la sensibilidad y de las condiciones físicas del individuo (pues un individuo que, por ejemplo, está enfermo difícilmente puede ejercitar la inteligencia).

Resulta claro, de todo lo visto hasta el momento, que el conocimiento intelectual es una actividad más perfecta que las vistas anteriormente, puesto que no tiene un límite en cuanto sí misma, sino en cuanto que está ligada a un cuerpo y necesita de percepciones sensibles para ejercerse. Por tanto, el placer concomitante a la actividad de entender es también más perfecto ${ }^{38}, \mathrm{y}$ ahora se trata de ver en qué medida.

En contraste con los movimientos procesuales del mundo está la contemplación, que propiamente no tiene lugar en el tiempo: al igual que la vista, uno piensa y ya ha pensado, es un acto único presente en un todo completo $^{39}$, pero a diferencia de ésta no depende de un órgano corporal ${ }^{40}$, por lo que se sitúa más allá de lo procesual, y es la actividad más perfecta. No obstante, esto es cierto sólo desde un punto de vista, porque, en el caso del ser humano, la actividad intelectual viene posibilitada por el conocimiento que previamente aporta la sensibilidad ${ }^{41}$ y porque en el caso de los hombres esta actividad viene precedida por el correcto desenvolvimiento de las facultades vitales más básicas ${ }^{42}$. Ciertamente, el ser humano no puede dedicarse a la contemplación hasta que no ha satisfecho las necesidades elementales ${ }^{43}$; sin embargo, una vez que se han satisfecho las necesidades básicas, la actividad que puede ejercer con más continuidad es la intelectual, porque de suyo no se encuentra vinculada a ningún órgano corporal, y por lo tanto no se agota. En el caso de Dios, su activi-

38 "Denn zur Lust gehören, wie schon gesagt wurde, drei Dinge: ein der Natur entsprechendes und geliebtes Gut, der Besitz dieses Gutes und die Erkenntnis dieses Besitzes. In allen diesen Beziehungen ist die geistige Lust viel größer und vollkommener als die sinnliche. Der erkannte Gegenstand steht viel höher, der Besitz ist viel vollkommener, weil der Verstand nicht bloß die äusern zufälligen Bestimmungen, sondern auch das Wesen der Dinge erkennt. Auch die Erkenntnis des Besitzes ist viel vollkommener". CATHREIN, V., Lust und Freude: Ihr Wesen und ihr sittlicher Charakter, mit besonderer Berücksichtigung der Lehre des Aristoteles, Felizian Rauch, Innsbruck 1931, p. 22.

${ }^{39}$ Cfr. Met. $\Theta .6,1048$ b 33-35.

40 "Sería ilógico que estuviese mezclado con el cuerpo, y es que en tal caso poseería alguna cualidad, sería frío o caliente y tendría un órgano como lo tiene la facultad sensitiva”. De Anima III.4, 429 a 24-27.

41 Cfr. De Anima III.7, 431 b 3-4.

42 Cfr. De Anima II.3, 415 a 2-8.

${ }^{43}$ Cfr. Eth. Nic. X.8, 1178 b 34-36: "el hombre contemplativo, por ser hombre, tendrá necesidad del bienestar externo, ya que nuestra naturaleza no se basta a sí misma para la contemplación, sino que necesita de la salud del cuerpo, del alimento y de los demás cuidados". 
dad de autoconocimiento no viene mediada por nada material o potencial $\mathrm{y}$, por lo tanto, posee una plenitud actual.

A este respecto, resulta importante considerar que la contemplación, como acto intelectual perfecto, no supone un momento de búsqueda, sino que es un acto simple, finalizado en sí mismo. El proceso de aprendizaje no puede ser, propiamente hablando, contemplación, sólo lo es el acto en el que la inteligencia posee la verdad, en que la contempla de manera directa, sin mediación de razonamientos. Aristóteles afirma que "es más agradable la existencia de los que saben que la de aquellos que buscan" 44 . El ejercicio mental de búsqueda de la verdad no es placentero, sino más bien costoso, e implica esfuerzo, mientras que la posesión de la verdad es simple y perfecta ${ }^{45}$.

\section{Objeto de la contemplación}

Hasta aquí llega lo que se puede decir de este tipo de acto, pero queda por ver cuál es su objeto. En el caso de Dios es evidente, pues él es objeto para sí mismo, y se conoce a sí mismo de manera plena y evidente en su único acto; sin embargo, en el caso del ser humano la cuestión no es tan sencilla, y es en este punto donde surgen las mayores divergencias. Quizás una de las cuestiones más discutidas sea la opinión de Ross, para el cual dentro de la contemplación entrarían las matemáticas, la metafísica y quizás la filosofía natural ${ }^{46}$. Sin embargo Defourny ${ }^{47}$, Leonard ${ }^{48}$ y

${ }^{44}$ Eth. Nic. X.7, 1177 a 27.

45 Sin embargo, es difícil dilucidar cómo es este acto, sobre todo en el caso del ser humano: según M. D. Philippe, la $\theta \varepsilon \omega \rho i ́ \alpha$, al ser una actividad perfecta, no puede identificarse con los actos particulares del voũ s: aprehensión, juicio y razonamiento. Se trataría de un acto simple por encima de éstos. Cfr. PhILIPPE, M. D., "Nature de l'acte de contemplation philosophique dans la perspective des principes d'Aristote", Revue Thomiste 49 (1949), p. 531. Se trata de un saber en acto por encima de las operaciones intelectuales: éstas suponen el camino hacia la verdad, pero la contemplación es el contacto directo con ella. En esta línea se podría situar la opinión de A. Kenny, para el cual la sabiduría no está tanto en la mera posesión de conocimientos como en la comprensión de esos conocimientos como verdaderos, entendiendo su posición real en el mundo. Cfr. KenNY, A., Aristotle on the Perfect Life, Clarendon Press, Oxford, 1995, p. 104. Contemplar equivale entonces a entender: captar lo qué es algo, en sí mismo y en relación al mundo, del mismo modo que R. Kraut pone énfasis en que contemplar no tiene tanto que ver con la búsqueda de conocimientos como con la reflexión que se hace sobre unos conocimientos ya adquiridos. Cfr. KRAUT, R., Aristotle on the Human Good, Princeton University Press, Princeton, 1989, p. 73.

${ }^{46}$ Cfr. Ross, W. D., Aristotle, Methuen \& Co, London, 1949, p. 234.

${ }^{47}$ Cfr. Defourny, P., 'L'activité de contemplation dans les Morales d'Aristote", Bulletin de l'Institut historique belge de Rome XVIII (1937). 
Gauthier ${ }^{49}$ se oponen a que la contemplación pueda ser algo distinto de la metafísica. Si la matemática es el estudio de los números -siendo estos una abstracción que hace la inteligencia-, y por filosofía natural entendemos la física -el estudio de los cuerpos del mundo en cuanto sujetos al cambio y al movimiento-, entonces resulta claro que estas disciplinas no se ocupan de comprender qué sean las cosas (contemplación) sino de algún aspecto concreto de la realidad ${ }^{50}$. Así, señalan estos autores que el objeto de la contemplación es la sustancia (pues entender qué es algo es entender su sustancia), y de modo más eminente la sustancia primera: Dios. Así, el acto de contemplación filosófica llega al ser de la sustancia primera, a su existencia ${ }^{51}$. Está opinión tiene fundamento en lo que dice Aristóteles al final de la Ética a Eudemo:

"Dado que el hombre consiste por naturaleza de una parte que manda y otra que obedece, y cada uno vivirá de acuerdo con el principio que manda en él, [...] de igual modo sucede con la capacidad contemplativa ( $\theta \varepsilon \omega \rho \varepsilon \tau \iota \kappa o ́ v)$. [...] De tal manera que aquel modo de elegir y de adquirir cosas buenas por naturaleza (bien sean corporales, riquezas, amigos y otros bienes) que permita una mejor contemplación de Dios ( criterio" 52 .

Aristóteles pone a Dios como el objeto de conocimiento más propio de la $\theta \varepsilon \omega \rho i ́ \alpha$, aquel que hace que esta actividad sea más perfecta y por lo tanto la más elevada en el hombre. Si se tiene además en cuenta que el placer en la actividad intelectual se da en la medida en que el objeto se adecua a la capacidad de conocer ${ }^{53}$ se podría admitir sin reservas que el placer mayor se daría en la contemplación de Dios.

En apoyo de esta tesis hay otro texto en la Metafísica:

48 Cfr. Leonard, J., Le Bonheur chez Aristote, Académie royale de Belgique, Bruxelles, 1948, p. 138.

${ }^{49}$ Cfr. Gauthier, R. A., La morale d'Aristote, Presses Universitaires de France, Paris, 1963, p. 101.

50 Hay un pasaje que daría pie a considerar la matemática como actividad contemplativa: "la función de la visión es el acto de ver, y de la ciencia matemática, la con-

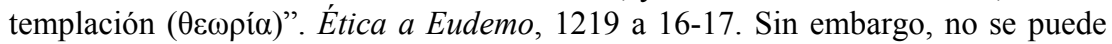
precisar el uso de $\theta \varepsilon \omega \rho i ́ \alpha$ en este contexto, y bien pudiera ser que Aristóteles quisiese decir "estudio" en vez de "contemplación".

${ }^{51}$ Cfr. Philippe, M. D., "Nature de l'acte de contemplation philosophique dans la perspective des principes d'Aristote", Revue Thomiste 49 (1949), p. 531.

52 Ética a Eudemo VII.15, 1249 b 9-19.

${ }^{53}$ Cfr. Eth. Nic. X.4, 1174 b 34-1175 a 2. 
"El entendimiento es movido por lo inteligible (voũ $\delta \dot{\varepsilon}$ ùं ò $\tau$ oũ

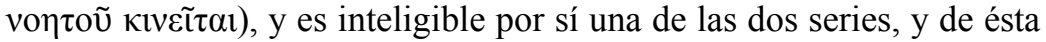
es la primera la sustancia, y de las sustancias, la que es simple y está en acto" ${ }^{54}$.

De aquí se puede deducir que el objeto más adecuado al entendimiento es la sustancia primera, y que es así como el hombre alcanza el más alto grado de contemplación y de placer. Pero, ¿es posible este conocimiento? No parece que el hombre pueda contemplar a Dios de manera directa, ya que éste se sitúa más allá del mundo sensible; por tanto, sólo es posible conocerlo por su presencia en el mundo, es decir, en cuanto causa final que mueve sin ser movido ${ }^{55}$. La propia sustancialidad de Dios se nos puede hacer presente (es decir, que podemos contemplarla) a través de la acción presente en las sustancias sensibles, que sí que están a nuestro alcance ya que se trata de la causalidad final que no puede separarse de la sustancia misma de su causa. Más que contemplar a Dios, el hombre lo que puede es contemplar la dependencia de las sustancias del mundo respecto a la sustancia primera, ver en los actos imperfectos, revestidos de potencialidad, un indicio del Acto Puro.

La comprensión del ente en cuanto ente corresponde únicamente a la filosofía ${ }^{56}$, y es en esta actividad en la que se da la contemplación en su sentido más propio. Una cuestión que queda abierta es cómo son posibles otros placeres intelectuales, como en las ciencias particulares o en el arte $y$ las actividades en las que entra en juego la inteligencia de manera menos intensa. Resulta claro que hay placeres en muchas actividades a nivel sensible-intelectual, y Aristóteles los cita a menudo: en la geometría, las artes y la arquitectura ${ }^{57}$; en la música y la conversación ${ }^{58}$; o en los juegos ${ }^{59}$. Sin embargo, Aristóteles no dice nada sobre si hay en estas actividades algún tipo de contemplación o en qué medida son actividades intelectuales ${ }^{60}$.

${ }^{54}$ Met. $\Lambda .7,1072$ a 30-32.

${ }^{55}$ Cfr. Met. $\Lambda .7,1072$ a 26-27.

${ }^{56}$ Cfr. Met. Г.1, 1003 a 21-26.

57 Cfr. Eth. Nic. X.5, 1175 a 35-37.

58 Cfr. Eth. Nic. X.5, 1175 b 4-6.

${ }^{59}$ Cfr. Eth. Nic. X.6, 1176 b 9.

${ }^{60}$ Burnet por su parte sugiere que dentro de la contemplación podrían entrar tanto la filosofía como la religión y el arte (cfr. BURnET, J., The Ethics of Aristotle, Metheuen \& Co, London 1900, p. 438), pero la mayoría de los comentadores se muestran disconformes, entre ellos Ross y Hardie, dado que no encontramos textos que den pie a justificar la experiencia estética como contemplación. Cfr. Ross, W. D., Aristotle, Methuen \& Co, London, 1949, p. 234; HARDIE, W. F. R., Aristotle's 


\section{6. ¿Es el placer intelectual una sensación?}

Según se ha visto, el placer intelectual es el más perfecto, pues corresponde a la actividad más perfecta y menos sujeta a la materialidad. En el caso de Dios es un acto sin potencialidad, sin impedimentos de ningún tipo; en el caso del hombre la acción contemplativa requiere de la sensación para que se lleve a cabo, pues se contempla a partir de lo que se conoce por los sentidos. Sin embargo, el acto intelectual parece ser algo que está más allá de la sensibilidad y, por otra parte, cabe plantearse si el placer intelectual es en sí mismo algo puramente intelectual o si depende de nuestra sensibilidad. El hombre contempla desde su cuerpo, ya que el cuerpo y el alma no se dan separados ${ }^{61}$. Por tanto, hay que admitir que algún tipo de experiencia sensorial acompaña la actividad contemplativa, y que en ella se da placer62. Sin embargo, en el caso del hombre la actividad intelectual y su placer correspondiente se da junto con la sensación porque, además, a la contemplación le acompaña frecuentemente la emoción, y ésta es un fenómeno psicosomático acompañado de placer.

No obstante, también hay textos del Estagirita que dan pie a pensar que el placer no es únicamente sensible-corporal, sino que puede darse al margen de nuestra materialidad. El caso más paradigmático es el de Dios, que es definido como acto puro, sin mezcla de potencialidad ${ }^{63}$, y del que

Ethical Theory, Clarendon Press, Oxford, 1980, p. 340. Por otro lado, la contemplación de Dios es un acto intelectual (de sabiduría) y no hay razón para incluir a la religión en éste. Cfr. VANIER, J., Le bonheur: principe et fin de la morale aristotélicienne, Desclée de Brouwer, Paris, 1965, p. 385.

${ }^{61}$ Ciertamente el entendimiento (voũ $\varsigma$ ) es la única facultad humana que se podría dar separada, debido a que su actividad no parece depender del cuerpo. Sin embargo, de hecho se da junto al cuerpo en la vida humana, y por tanto su actividad se da junto con las sensaciones y las emociones: "el entender (voعĩv), parece algo particularmente exclusivo del alma; pero ni esto siquiera podrá tener lugar sin el cuerpo si es que se trata de un cierto tipo de imaginación o de algo que no se da sin imaginación". De Anima I.1, 403 a 8-10. "Más adelante, por lo demás, habremos de examinar si el intelecto puede o no entender (vociv) algo que exista separado de la

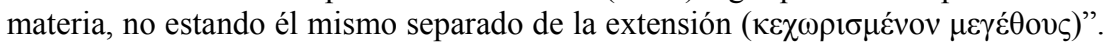
De Anima III.7, 431 b 18-19.

62 "Les plaisirs de la pensée sont eux-mêmes accompagnés d'une sensation physique: nous avons chaud ou froid, nous rougissons ou nous palissons sous l'influence de nos propres idées. [...] Le plaisir et la peine semblent donc être des affections communes à l'âme et au corps, comme la sensation, la mémoire, la passion, $\theta 0 \mu$ óc, le désir sensuel, on pourrait dire même la pensée, puisque la pensée est accompagnée presque nécessairement d'une image, et que l'imagination est liée à la sensation". Chaignet, A., Essai sur la psychologie d'Aristote, Culture et civilization, Bruxelles, 1966, (Paris, 1883), p. 443.

${ }^{63}$ Cfr. Met. $\Lambda .6,1071$ b 12-20. 


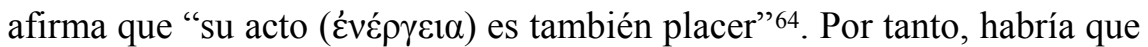
admitir que es posible un placer puramente intelectual ${ }^{65}$ (pues la actividad pura de Dios es entendimiento), separado de cualquier tipo de corporalidad; el placer contemplativo humano debe ser semejante a éste, un placer que acompaña a un sentir "anímico", no corporal, aunque no sea sencillo saber dónde termina el placer sensible y dónde empieza el estrictamente intelectual, dada la índole psicosomática del ser humano.

De todo esto, resulta que el placer no puede ser considerado como una "sensación recibida" o un elemento pasivo, puesto que no tendría sentido que Dios, acto puro, autoconocimiento sin mezcla de potencialidad, pueda "ser afectado". El placer, al menos en el caso de Dios, es algo inherente a la propia actividad de contemplación, un "sentirse" sin impedimentos, sin potencialidad. Además, se trata de un "sentirse" puramente espiritual, sin mediación material. El placer, por tanto, tiene que ver sobre

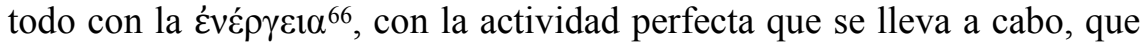
en último término es la autoconciencia de plenitud:

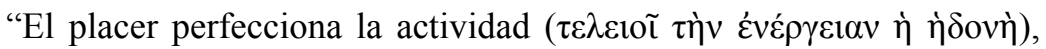
no como la disposición que le es inherente, sino como cierta

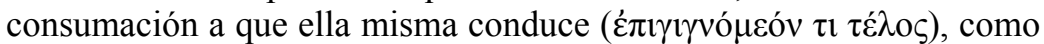
la juventud a la flor de la vida. Por consiguiente, siempre que lo que se piensa o se percibe por los sentidos sea como debe, y lo sea igualmente la facultad que juzga o contempla, se dará en la actividad

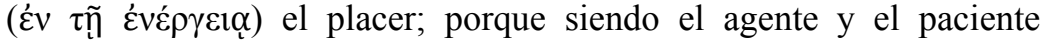
semejantes, y estando dispuestos el uno hacia el otro de la misma manera, se produce naturalmente el mismo efecto" 67 .

${ }^{64}$ Met. $\Lambda .7,1072$ b 16.

65 Hay pasajes en los que parece que Aristóteles liga el placer estrictamente a la sensibilidad (De Anima III.7, 431 a 10-12) y, en cierto sentido, todo placer es un "sentirse". Por tanto, parece que para poder admitir un placer intelectual es necesario admitir un tipo de "sensación espiritual": "The admission of pure pleasures of the speculative intellect would seem to imply an intellective or spiritual feeling. Sometimes, however, Aristotle insists on the relation of all pleasure to bodily sensation. For pleasure, he says, is either in present action, in which case it is a direct sensible experience excited by a sensibly perceived object, or in memory or anticipation, which are dependent upon that experience. [...] But besides sensuous conation Aristotle recognizes a conation of the rational will. In the contemplation of an intelligible object the mind is affected by a spiritual pleasure inseparable from a spiritual conation". GARDINER, H. N., "The Psychology of the Affections in Plato and Aristotle", The Philosophical Review 28 (1919), pp. 11-12.

${ }^{66}$ Cfr. Bostock, D., "Pleasure and Activity in Aristotle's Ethics", Phronesis 33 (1988), p. 269.

${ }^{67}$ Eth. Nic. X.4, 1174 b 32-1175 a 4. 


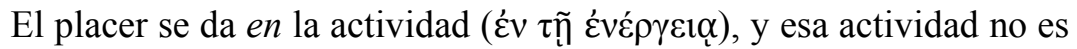
otra que la cognoscitiva. El placer es la autoexperimentación satisfactoria del sujeto cuando las actividades conscientes (sentir y pensar) encuentran su objeto adecuado ${ }^{68} \mathrm{y}$, a la vez, no es algo extraño a esas actividades de conocimiento, sino que las perfecciona "desde dentro" 69 . De este modo, el placer parece situarse más allá de la corporalidad, de lo potencial (aun-

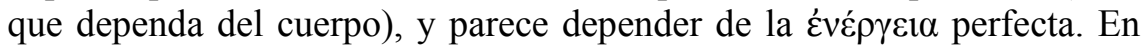
este sentido, si se considera el placer como una "sensación", como una "autoexperimentación", debe ser -al menos en el caso del placer intelectual- algo que va más allá de lo corporal.

\section{Conclusiones}

La consideración de varios textos de Aristóteles acerca de la contemplación humana y el placer divino ha mostrado que es posible pensar que el placer no es únicamente sensible-corporal, sino que puede darse al margen de nuestra materialidad. Esta suposición encaja con la doctrina del placer del libro X de la Ética a Nicómaco, en la que Aristóteles vincula el placer con la actividad perfecta ( $v^{\prime} \varepsilon \dot{\rho} \rho \gamma \varepsilon 1 \alpha \tau \varepsilon \lambda \varepsilon^{\prime} \alpha$ ). Evidentemente el caso más paradigmático de actividad perfecta es de Dios, que es definido como acto puro, sin mezcla de potencialidad, y del que afirma que su acto

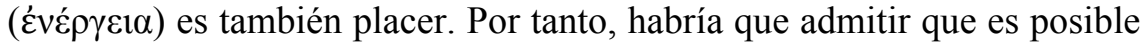
un placer puramente intelectual (pues la actividad pura de Dios es entendimiento), separado de cualquier tipo de corporalidad. El placer contem-

68 "Aristotle's account of pleasure has occasioned unusually strident responses from his detractors: they find his view incredible, incomprehensible, circular. He babbles. By reflecting on the metaphysics of pleasure in Nicomachean Ethics X, we can appreciate that whatever its ultimate merits, Aristotle's view is neither incomprehensible nor incredible. He thinks that pleasures arise in the activities of perception and thought, when our faculties are functioning at their highest level and arrayed over their finest objects. He thinks, further, that pleasures in turn perfect or complete those same activities: pleasure contributes to such perceptions and thoughts by making them what they are, namely the highest forms of human cognition. A consummate thought or perception, thinks Aristotle, is a pleasurable thought or instance of perception. This seems not only comprehensible, but credible and even defensible: we are not subjects who experience pleasures independently of our thinking or perceiving. We are rather subjects who experience pleasure in our thinking and our perceiving -precisely when our faculties are functioning at their finest". SHIELDS, C., "Perfecting Pleasures: The Metaphysics of Pleasure in Nicomachean Ethics X", Aristotle's Nicomachean Ethics. A Critical Guide, J. Miller (ed.), Cambridge University Press, Cambridge, 2011, pp. 209-210.

${ }^{69}$ Ricken, F., "Wert und Wesen der Lust", Aristoteles. Die Nikomachische Ethik, O. Höffe (ed.), Akademie Verlag, Berlin, 1995, pp. 226-227. 
plativo, en el caso del ser humano, debe ser semejante a éste, un placer que acompaña a un sentir "anímico", no corporal, aunque no sea sencillo saber dónde termina el placer sensible y dónde empieza el estrictamente intelectual, dada la índole psicosomática del ser humano.

\title{
RESUMEN:
}

Aristóteles afirma en la Ética a Nicómaco que el placer acompaña a la actividad perfecta, haciéndola más deseable, y que la actividad contemplativa es la más divina y placentera. Por otro lado, en la Metafísica dice que la actividad de Dios es sumo placer, porque su actividad es perfecta. En este artículo trato de estudiar la conexión entre placer y actividad en la contemplación y su relación con la actividad de Dios, para poder entender el agrado intelectual: ¿es posible hablar de un placer "espiritual”? ¿Hay sensaciones desligadas de los órganos materiales?

Palabras clave: Actividad, Aristóteles, Dios, Eudoxo, sensación, placer.

\begin{abstract}
:
Aristotle says in the Nicomachean Ethics that pleasure accompanies the perfect activity, doing it more desirable, and that the activity of contemplation is the most divine and pleasurable. On the other hand, he says in the Metaphysics that God's activity is the most pleasurable, because his activity is perfect. In this article I try to study the relation between pleasure and activity in contemplation and its relation with God's activity, in order to understand intellectual pleasure: is it possible to speak about a "spiritual" pleasure? Are there sensations which do not depend on material organs?
\end{abstract}

Key words: Activity, Aristotle, Eudoxus, God, Sensation, Pleasure. 